Ocular Oncology

and Pathology
Ocul Oncol Pathol 2016;2:202-205

DOI: $10.1159 / 000444260$
Received: October 5, 2015

Accepted after revision: January 25, 2016

Published online: March 10, 2016

\title{
Polarizable Material from a Conjunctival Biopsy in a Patient with Sarcoidosis
}

\author{
Omair B. Alia Ryan Deroque a R. Grant Morshedi ${ }^{a}$ Harry Brown ${ }^{a}$ b \\ Departments of a Ophthalmology and ${ }^{b}$ Pathology, University of Arkansas for Medical Sciences, Little Rock, Ark., USA
}

\begin{abstract}
Key Words
Conjunctiva - Uveitis glaucoma - Sarcoidosis - Foreign body . Polarizable material $\cdot$ Conjunctival biopsy
\end{abstract}

\begin{abstract}
Purpose: Conjunctival biopsies may contain polarizable material in patients with sarcoidosis despite no history of prior trauma or eye surgeries. Procedures: A 39-year-old male with uveitic glaucoma presented with decreased vision and throbbing pain in his right eye. His intraocular pressure was elevated, and his vision was reduced to hand motion. Due to persistently elevated intraocular pressure refractory to medical treatment, the patient underwent a glaucoma drainage device procedure. During the procedure, a yellow, nodular conjunctival growth was noted and biopsied. Results: Histopathological examination revealed multiple nonnecrotizing granulomata, some of which contained polarizable material. Conclusions: Biopsies of patients with sarcoidosis may contain polarizable material without evidence of foreign body inoculation.

(c) 2016 S. Karger AG, Basel
\end{abstract}

\section{Introduction}

Sarcoidosis is a systemic disease of unknown etiology characterized pathologically by the formation of nonnecrotizing granulomata consisting of aggregates of epithe- lioid histiocytes (often with multinucleated giant-cell formation) and CD4+ lymphocytes [1]. African-Americans are more commonly and more severely affected than other races [2]. Typically, patients with sarcoidosis are young adults and present with bilateral hilar lymphadenopathy, pulmonary infiltrates, and skin, joint and/ or eye lesions. The exact etiology remains undetermined; however, the prevailing theory is that genetically susceptible hosts are exposed to an unknown environmental antigen, which triggers a Th1-type immune response [1]. This results in the formation of the characteristic granulomata. The organs most frequently affected are the lungs, skin and eyes, which supports the view of sarcoid granulomatous inflammation as a response to an environmental agent. The diagnosis of sarcoidosis is made by correlating clinical presentations with radiologic and histopathological findings and by excluding other identifiable causes of granuloma formation. Due to the variability in presentation, a tissue biopsy is often performed in order to confirm the presence of granulomata and to rule out other causes of granuloma formation. Material within granuloma, which is highlighted under polarized light in histological sections from patients with sarcoidosis, has long been attributed to foreign body inoculation [3]. We report a case of a male patient with clinical, radiographic and laboratory findings consistent with sarcoidosis, who underwent a conjunctival biopsy that contained polarizable material. This patient had no history of exposure to exogenous material.

\section{KARGER}

E-Mail karger@karger.com

www.karger.com/oop
(C) 2016 S. Karger AG, Basel

2296-4681/16/0023-0202\$39.50/0
Omair B. Ali

Jones Eye Institute

4110 Outpatient Circle \#523

Little Rock, AK 72205 (USA)

E-Mail oali@uams.edu 


\section{Patient Presentation}

A 39-year-old African-American male presented with a 5-day history of decreased vision, photophobia and severe, throbbing pain in his right eye (OD). The patient denied recent trauma or exposure to environmental irritants. Past medical history included systemic hypertension and anterior uveitis. The patient was given a presumptive diagnosis of sarcoidosis based on previous laboratory tests and clinical correlation. He had no other clinical manifestations aside from uveitis. Two years ago, investigations for uveitis revealed a normal chest X-ray, an elevated serum lysozyme and a positive antinuclear antibody. Because of the lack of specificity of these results, the diagnosis of sarcoidosis was never confirmed. On current ophthalmological examination, visual acuity was hand motion OD. Biomicroscopy revealed conjunctival and scleral injection, and folds in Descemet's membrane. There were $2+$ anterior chamber cells and posterior synechiae resulting in a sluggish pupillary response to light. Applanation tonometry OD revealed an intraocular pressure of $43 \mathrm{~mm} \mathrm{Hg}$. Gonioscopy revealed multiple large, broad-based peripheral anterior synechiae in all quadrants. Evaluation of the optic nerve head showed a cup:disc ratio of 0.9. Dilated funduscopy OD was not performed due to synechial angle closure and elevated intraocular pressure. Slit-lamp, dilated and gonioscopic examinations were unremarkable in the left eye (OS).

Due to the elevated intraocular pressure refractory to medical therapy, the patient underwent an Ahmed valve implant (New World Medical, Inc., Rancho Cucamonga, Calif., USA) OD. Intraoperatively, an area of yellowish, thickened tissue was noted in the inferior bulbar conjunctiva and an incisional biopsy was taken (fig. 1). At followup 2 weeks after the surgery, visual acuity was unchanged, but intraocular pressure was reduced to $18 \mathrm{~mm} \mathrm{Hg} \mathrm{OD}$ and $10 \mathrm{~mm} \mathrm{Hg}$ OS.

\section{Ancillary Tests}

Serological examination revealed an unremarkable complete blood count and a basic metabolic panel. No infectious etiologies were detected on rapid plasma reagin, fluorescent treponemal antibody absorption and tuberculosis-specific Enzyme-Linked ImmunoSpot (TSPOT) assays. Serum angiotensin-converting enzyme was $152 \mathrm{U} / \mathrm{l}$ (normal range, 8-55) and lysozyme was $>32$ $\mu \mathrm{g} / \mathrm{ml}$ (normal range, 2.7-9.4). A chest radiograph showed scarring in the left upper lobe with no signs of tuberculosis, pulmonary congestion or hilar lymphadenopathy.

Polarizable Material in Conjunctiva Sarcoidosis

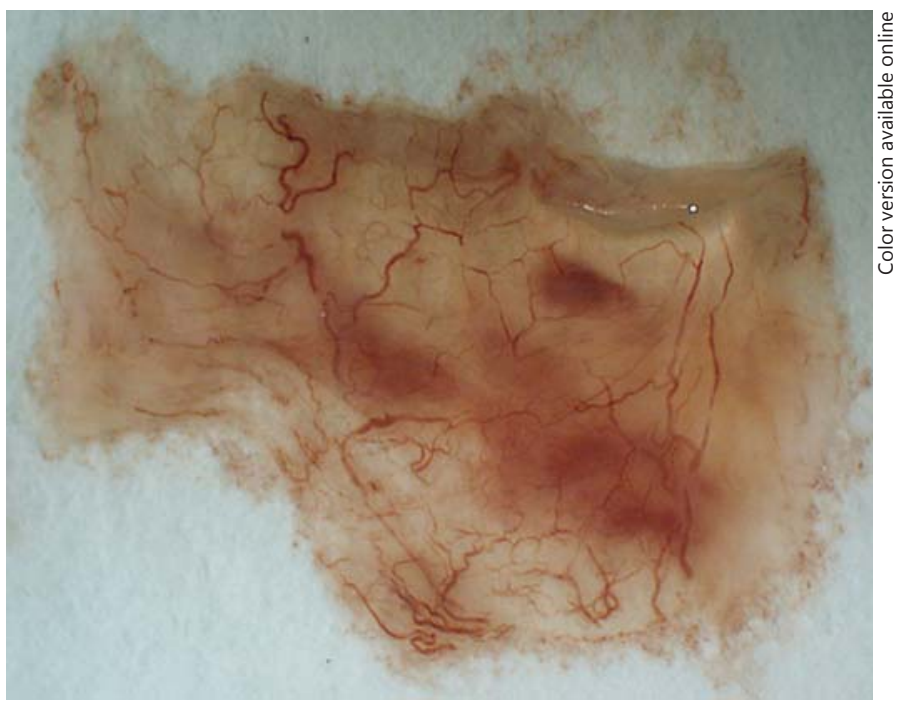

Fig. 1. Gross specimen of the conjunctival biopsy. Gross appearance of the conjunctival tissue. No nodules are apparent (original magnification $\times 10$ ).

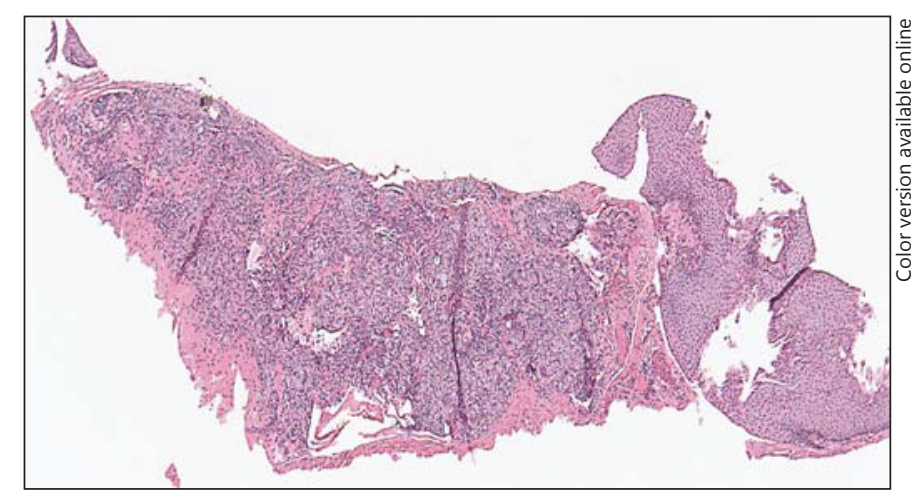

Fig. 2. H\&E stain of the biopsy specimen. Low-power photomicrograph of the conjunctival biopsy specimen, showing multiple granulomata involving conjunctival stroma. Note the relative absence of surrounding chronic inflammatory cells ('naked' granulomata; original magnification $\times 10$ ).

\section{Pathological Examination}

Histopathological examination of formalin-fixed tissue demonstrated conjunctival tissue with unremarkable epithelium overlying stroma containing multiple small nongranulomata (fig. 2), some of which contained nonuniform, variably sized, rounded-to-angular polarizable material lacking features of calcium oxalate (fig. 3a, b). No fungi or acid-fast bacilli were identified on Gomori Methenamine Silver or acid-fast bacilli histochemical stains. 

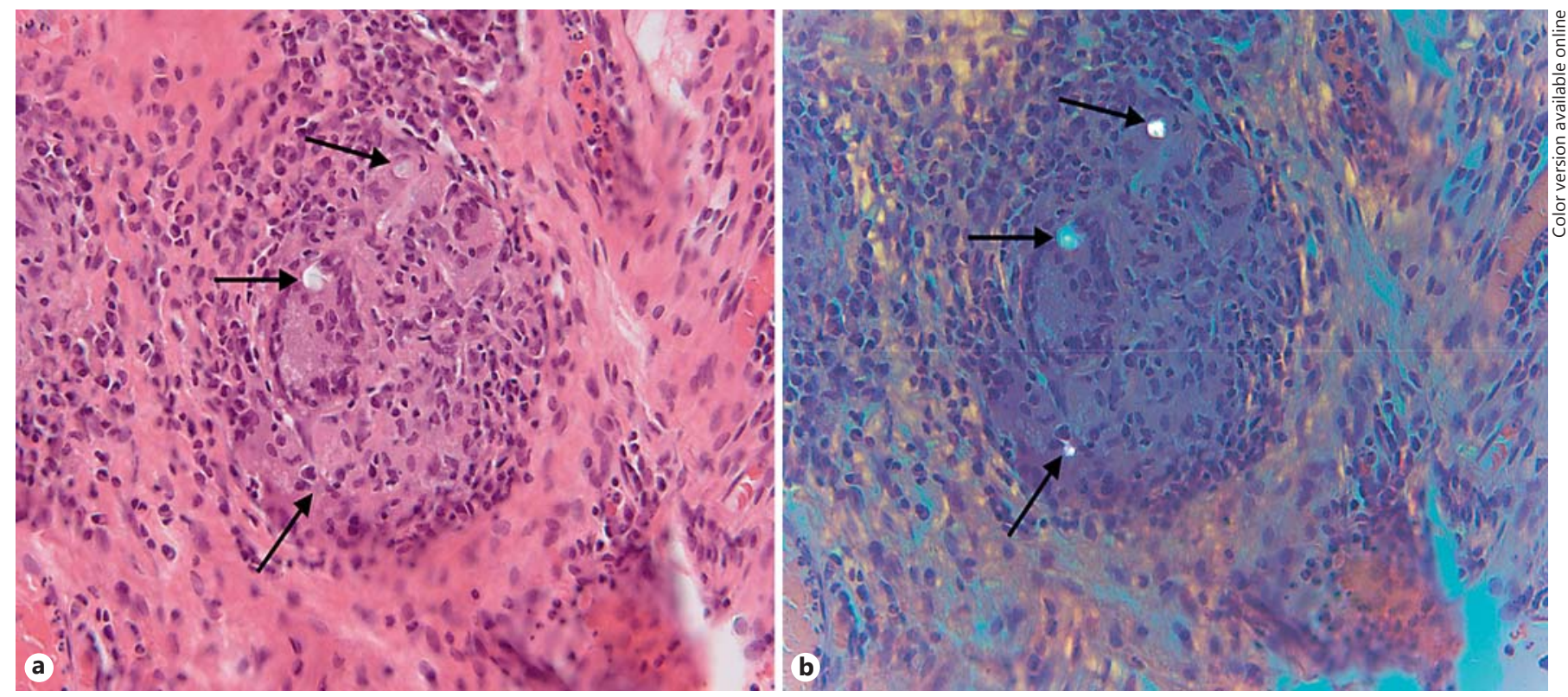

Fig. 3. a, b H\&E stain of the biopsy specimen with higher magnification under polarized light. Photomicrograph of nonnecrotizing granuloma. Multinucleated giant cells contain refractile materials (arrows; a), which are polarizable (b; original magnification $\times 400)$.

\section{Discussion}

Sarcoidosis is diagnosed by assessing clinical and radiologic findings and excluding other causes of granuloma formation. A diagnosis is best supported by supplementing the clinical picture with histological evidence of tightly packed, noncaseating granulomata usually bordered by lamellar hyaline collagen. Although there is no pathognomonic feature in sarcoidosis, there are a variety of intracytoplasmic inclusions commonly seen in biopsy specimens. These include birefringent calcium oxalate crystals, cholesterol crystals, star-shaped cytoplasmic inclusions called asteroid bodies, and concentrically lamellated calcified nodules within the cytoplasm of giant, multinucleated cells called Schaumann bodies [4].

Eyes and adnexal structures are frequently involved and may often be the presenting symptom in sarcoidosis. Ocular symptoms may include dry eye, blurry vision, photophobia, redness and pain with resultant diagnoses including uveitis, keratoconjunctivits sicca and adnexal granulomata [5]. Anterior uveitis is the most common ocular comorbidity seen in patients with sarcoidosis [5]. Biopsies of lesions on conjunctivae have proven to be helpful because pulmonary biopsies carry more risk and are more invasive. Even in the absence of visible conjunctival granulomata, a conjunctival biopsy is necessary for the diagnosis of definite ocular sarcoidosis [6]. A tissue sample is integral in a diagnosis of sarcoidosis to identify the granulomatous reaction and to rule out other causes of similar inflammation.

Polarizable material found in cutaneous biopsies taken from patients with sarcoidosis attributed to a sarcoid granulomatous reaction to an inoculated foreign body. Marcoval et al. [7] reported that $22 \%$ of the cutaneous biopsies of patients with sarcoidosis contain exogenous material when viewed under polarized light. Those samples without visible foreign bodies were suspected to have had previous imperceptible inoculation of a foreign body now no longer present in the tissue $[3,7]$. The majority of cutaneous biopsies are taken from locations of frequent minor, undetected trauma, such as knees and elbows. Thus, it has been inferred that the presence of polarizable material is due to a sarcoid-like granulomatous reaction to exogenous material [7]. Our patient, however, reported no ocular trauma, had no previous ocular surgical history, and had no evidence of inoculated foreign material in the biopsy. Moreover, it is unlikely that exogenous material was implanted in the inferior bulbar conjunctiva without the patient perceiving it. Polarizable materials seen in the tissue sample may be intracytoplasmic inclusions in multinucleated, giant cells. Metabolism of sarcoid granulomata by macrophages yields many distinct, 
endogenous by-products, some of which may be visible under polarized light and are often misidentified as exogenous material [4]. It is also possible that the polarizable material is an endogenous by-product acting as an inciting agent for the development of sarcoid granulomata.

The diagnosis of sarcoidosis cannot be made solely on biopsy because there are many granulomatous disorders with a similar histopathological appearance; the diagnosis must integrate clinical and radiological evidence with histological findings. Overall assessment of our patient strongly supports the diagnosis of sarcoidosis, even though it is an atypical presentation. Elevated serum angiotensin-converting enzyme and lysozyme, although not specific findings of sarcoidosis, are suggestive of sarcoidosis and aid in narrowing the differential diagnosis [8]. These results in a young, African-American male, coupled with recurrent anterior uveitis, positive antinuclear antibody, and conjunctival nodules with histopatholocial evidence of nonnecrotizing granulomata, strongly support a diagnosis of sarcoidosis. The presence of polarizable material in a conjunctival granuloma without a history of exogenous material in the eye, however, raises the question that perhaps other mechanisms yielding polarizable material exist and can be present in sarcoid granulomata. Considering the extreme variability of the presentation of sarcoidosis and the wide array of inclusions reported in giant cells, material visible under polarized light in tissue samples may be the by-product of an endogenous process of metabolism by macrophages.
Moreover, these findings are not necessarily indicative of a foreign body granuloma.

Case series describing the presence of polarizable material in cutaneous and pulmonary biopsies have been documented; however, none note the presence of polarizable material in a conjunctival biopsy in patients with sarcoidosis [3, 7]. The presence of exogenous material within sarcoid granulomata requires further investigation to better understand why it is present and how it relates to the pathogenesis of sarcoidosis. Physicians analyzing the presence of polarizable material in tissue samples of patients with sarcoidosis should consider various potential mechanisms along with foreign body inoculation. Furthermore, conjunctival biopsy is a valuable diagnostic tool and should be utilized even with an atypical presentation of sarcoidosis.

\section{Statement of Ethics}

The authors confirm that the subjects have given their informed consent, and the Committee on Human Research of the University of Arkansas for Medical Sciences has approved the study protocol.

\section{Disclosure Statement}

The authors declare that there are no conflicts of interest. Furthermore, there are no financial interests.

\section{References}

1 Spagnolo P: Sarcoidosis: a critical review of history and milestones. Clin Rev Allergy Immunol 2015;49:1-5.

2 Judson MA, Boan AD, Lackland DT: The clinical course of sarcoidosis: presentation, diagnosis, and treatment in a large white and black cohort in the United States. Sarcoidosis Vasc Diffuse Lung Dis 2012;29:119-127.

3 Walsh NM, Hanly JG, Tremaine R, Murray S: Cutaneous sarcoidosis and foreign bodies. Am J Dermatopathol 1993;15:203-207.
4 Rossi G, Cavazza A, Colby TV: Pathology of sarcoidosis. Clin Rev Allergy Immunol 2015; 49:36-44.

5 Birnbaum AD, French DD, Mirsaeidi M, Wehrli S: Sarcoidosis in the national veteran population: association of ocular inflammation and mortality. Ophthalmology 2015;122: 934-938.

6 Herbort CP, Rao NA, Mochizuki M: International criteria for the diagnosis of ocular sarcoidosis: results of the first International
Workshop on Ocular Sarcoidosis (IWOS). Ocul Immunol Inflamm 2009;17:160-169.

7 Marcoval J, Mañá J, Moreno A, Gallego I, Fortuño Y, Peyrí J: Foreign bodies in granulomatous cutaneous lesions of patients with systemic sarcoidosis. Arch Dermatol 2001;137:427-430.

8 Turton CW, Grundy E, Firth G, Mitchell D, Rigden BG, Turner-Warwick M: Value of measuring serum angiotensin I converting enzyme and serum lysozyme in the management of sarcoidosis. Thorax 1979;34:57-62. 